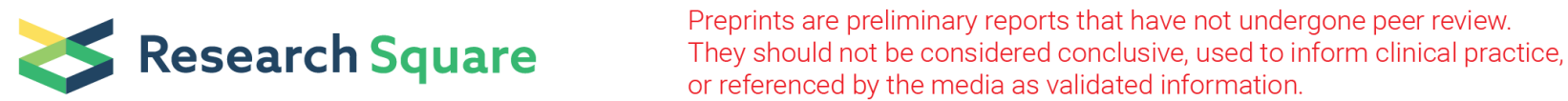

\section{Irradiated Fibroblasts Increase Interleukin-6 \\ Expression and Induce Migration of Head and Neck Squamous Cell Carcinoma}

Shinsuke Suzuki ( $\square$ suzukis@med.akita-u.ac.jp )

Akita University Graduate School of Medicine

Satoshi Toyoma

Akita University Graduate School of Medicine

Yohei Kawasaki

Akita University Graduate School of Medicine

Takechiyo Yamada

Akita University Graduate School of Medicine

\section{Research Article}

Keywords: Fibroblasts, head and neck squamous cell carcinoma, interleukin-6, irradiation, migration.

Posted Date: September 7th, 2021

DOI: https://doi.org/10.21203/rs.3.rs-849275/v1

License: (9) This work is licensed under a Creative Commons Attribution 4.0 International License.

Read Full License

Version of Record: A version of this preprint was published at PLOS ONE on January 28th, 2022. See the published version at https://doi.org/10.1371/journal.pone.0262549. 


\section{Abstract \\ Background}

The cytotoxic effect of radiation plays an important role in the treatment of head and neck cancer. However, irradiation is known to lead to the migration of various cancer cells, including those of head and neck cancer. Recently, fibroblasts in the cancer microenvironment have been reported to be involved in this mechanism. Nevertheless, the mechanism underlying the migration of head and neck cancer cells remains unclear. The purpose of this study was to elucidate this migration mechanism induced by irradiation in terms of the interaction of head and neck cancer cells with fibroblasts.

\section{Methods}

We used the head and neck squamous cell carcinoma (HNSCC) cell lines SAS and FaDu as well as fibroblast cell lines. These cells were irradiated and their viability was compared. In fibroblasts, changes in interleukin-6 (IL-6) secretion caused by irradiation were measured by enzyme-linked immunosorbent assay (ELISA). The cell migration ability of cancer cells was evaluated via a migration assay using a semipermeable membrane. HNSCC cells were cocultured with irradiated and nonirradiated fibroblasts, and their migration ability under each condition was compared. We also examined the effect of IL- 6 on the migration of HNSCC cells. Furthermore, to investigate the effect of fibroblast-derived IL- 6 on the migration ability of HNSCC cells, we conducted a coculture study using IL-6 neutralizing antibody.

\section{Results}

Irradiation reduced the survival of HNSCC cells, whereas fibroblasts were resistant to irradiation. Irradiation also increased IL- 6 secretion by fibroblasts. Migration of HNSCC cells was enhanced by coculture with fibroblasts and further enhanced by coculture with irradiated fibroblasts. We also confirmed that the migration of HNSCC cells was induced by IL- 6 . The enhanced migration of cancer cells caused by coculturing with fibroblasts was canceled by the IL-6 neutralizing antibody.

\section{Conclusion}

These results show that fibroblasts survive irradiation and induce the migration ability of HNSCC cells through increased secretion of IL-6.

\section{Background}

Radiotherapy plays an important role in the treatment of head and neck cancer; indeed, it has been used at every stage of treatment [1,2]. However, although irradiation has cytotoxic effects on cancer cells, it reportedly promotes tumorigenicity, and especially migration of cancer cells. This phenomenon results in 
increased local invasion, distant metastasis, and therefore poor prognosis for patients with various cancers including head and neck cancer $[3,4]$. Thus, the conflicting effects of irradiation on cancer cells are a major challenge to be overcome in cancer treatment.

In recent years, it has been found that irradiation induces tumorigenic effects not only in cancer cells but also the cancer microenvironment. This alteration in the cancer microenvironment is reportedly involved in the recurrence and metastasis of cancer after radiotherapy $[5,6]$. Mechanistically, the importance of various cytokines and growth factors in mediating the interaction between cancer cells and supporting cells, such as fibroblasts, in the cancer microenvironment has been confirmed [7-9]. In particular, interleukin-6 (IL-6) is known to be involved in the interaction between fibroblasts and cancer cells in the irradiated cancer microenvironment, and its role has attracted attention $[10,11]$.

To date, few studies have been conducted on irradiation-induced tumor progression in head and neck cancer; thus, much remains to be elucidated. The purpose of this study was therefore to elucidate the mechanism of irradiation-induced head and neck cancer cell migration in terms of the interaction of these cells with fibroblasts.

\section{Methods}

\section{Cells and cell culture}

We purchased SAS, a human tongue squamous cell carcinoma cell line, from the RIKEN Cell Bank (Tsukuba, Japan). FaDu, the cells from a human hypopharyngeal SCC cell line, were kindly gifted by the Department of Cell Biology and Morphology, Akita University Graduate School of Medicine (Akita, Japan). SF-TY, a human fibroblast cell line, was obtained from the Japanese Collection of Research Bioresources Cell Bank (JCRB Cell Bank, Osaka, Japan).

All cells were maintained in the Dulbecco's modified Eagle's medium (DMEM; Merck KGaA, Darmstadt, Germany) supplemented with $10 \%$ fetal bovine serum in a humidified atmosphere containing $5 \% \mathrm{CO}_{2}$ at $37^{\circ} \mathrm{C}$. For neutralization of IL-6, neutralizing mouse anti-IL-6 mAb (MAB406) and isotype control immunoglobulin G1 (IgG1) mAb (MAB005) were purchased from R \& D Systems (Minneapolis, MN)

\section{Irradiation}

Cells were seeded in $60 \mathrm{~mm}$ dishes and cultured to $80 \%$ confluence before being irradiated at 2, 5 , or 10 Gy at room temperature using a $160 \mathrm{kVp}$ cabinet X-ray system filtered with $0.5 \mathrm{~mm}$ Cu by the Faxitron CP160 (Faxitron X-Ray Corp., Wheeling, IL, USA). After irradiation, cells were cultured for $24 \mathrm{~h}$ prior to being harvested and subjected to an IL-6 enzyme-linked immunosorbent assay (ELISA) assay or cell migration assay. To conduct a cell survival assay, cells were cultured for 6 days after irradiation before being fixed and stained with crystal violet and then being observed under a light microscope (Olympus, Tokyo, Japan). 


\section{Cell survival assay (crystal violet staining)}

The cells were fixed in $4 \%$ paraformaldehyde for $20 \mathrm{~min}$ at room temperature and then stained with $0.04 \%$ crystal violet in $1 \%$ ethanol (20 min at room temperature). The plates were subsequently washed extensively under running tap water and air dried. After solubilization of the samples in $1 \%$ sodium dodecyl sulfate (SDS), the optical density values were read by plate reader at $550 \mathrm{~nm}$.

\section{Migration assay}

We evaluated cell migration in vitro using semipermeable modified Boyden inserts with a pore size of 8 $\mu \mathrm{m}$ (Becton, Dickinson and Company, Franklin Lakes, NJ, USA). In total, $3 \times 10^{4}$ SAS and FaDu cells were plated in the inserts. For the coculture assay, $5 \times 10^{4}$ fibroblast cells were plated in a holding well. Plating was conducted on serum-free DMEM. We plated the same number of cells from the inserts in 96-well plates to serve as loading controls. Both the inserts and holding wells were filled with serum-free medium composition. Depending on the needs of experiment, $10 \mathrm{ng} / \mathrm{ml}$ of IL-6, $20 \mathrm{ng} / \mathrm{ml}$ of IL-6 neutralizing antibody, or $20 \mathrm{ng} / \mathrm{ml}$ of control IgG were added to the medium. After $24 \mathrm{~h}$ of treatment at $37^{\circ} \mathrm{C}$ in a $5 \%$ $\mathrm{CO}_{2}$ incubator, we gently wiped away the cells in the insert using a cotton swab. Cells on the reverse side of the insert were fixed and stained with Diff-Quik ${ }^{\circledR}$ (Sysmex, Kobe, Japan) according to the manufacturer's instructions. We counted the invading cells in four representative fields using light microscopy at a magnification of 200x. Cells plated in 96-well plates were subjected to 3-(4,5dimethylthiazol-2-yl)-2,5-diphenyltetrazolium bromide assays and we normalized the cell numbers across the groups. We also adjusted the number of migrating cells accordingly.

\section{Western blotting}

We detected protein expression using western blot analysis with actin used as an internal control. We lysed cell lines in detergent containing 1\% NP-40, $150 \mathrm{mmol} / \mathrm{I} \mathrm{NaCl}, 1 \mathrm{mmol} / \mathrm{EDTA}, 0.1 \mathrm{mmol} / \mathrm{I}$ phenylmethylsulfonyl fluoride, $1 \mu \mathrm{g} / \mathrm{ml}$ leupeptin, and $1 \mu \mathrm{g} / \mathrm{ml}$ aprotinin and then determined the protein levels using the Bio-Rad Protein Assay method (Bio-Rad Laboratories Inc., Hercules, CA, USA). We separated $40 \mu \mathrm{g}$ of the total protein on $8 \%$ SDS-PAGE gels and transferred these to nitrocellulose membranes using a semidry transfer machine (Bio-Rad Laboratories). Next, we blocked membranes with $5 \%$ skimmed milk/TBS with Tween-20 solution for $1 \mathrm{~h}$ at room temperature, before incubating with primary antibodies in $5 \%$ skimmed milk in TBS-T overnight at $4^{\circ} \mathrm{C}$. After washing with TBS-T three times, we incubated the membranes for $1 \mathrm{~h}$ with horseradish-peroxidase-conjugated secondary antibody (BioRad Laboratories) at 1:3,000 diluted in 5\% skimmed milk in TBS-T. We then rinsed the filters with TBS-T three times and developed the blot using Luminol Reagent (Santa Cruz Biotechnology, Santa Cruz, CA, USA) by autoradiography. The band intensities were analyzed using ImageJ (U. S. National Institutes of Health). We used the following primary antibodies: mouse anti-IL-6Ra (1:1,000; Santa Cruz Biotechnology, Santa Cruz, USA), mouse anti-gp130 (1:1,000; Santa Cruz Biotechnology), and mouse anti-actin (1:3,000; Santa Cruz Biotechnology).

\section{ELISA}


The serum levels of IL-6 were measured by an ELISA using an ELISA kit (Proteintech, Rosemont, IL, USA.). To evaluate changes in IL-6 expression by irradiation in fibroblasts, $3 \times 10^{6}$ fibroblasts were seeded on 6well plates in DMEM with $10 \%$ fetal bovine serum (FBS) overnight. The medium was changed to serumfree DMEM and irradiated at $10 \mathrm{~Gy}$. Nonirradiated fibroblasts were also prepared as a control. At $24 \mathrm{~h}$ after irradiation, supernatants were collected, and subjected to an ELISA assay.

\section{Statistical analysis}

Statistical analyses were performed using Statcel 3 (OMS Publishing, Tokorozawa, Japan). A WilcoxonMann-Whitney two-tailed exact test was used to assess the statistically significant differences in cell survival, migration studies, and IL- 6 expression. Data are presented as means \pm standard deviation (SD) from experiments that were repeated at least three times. We considered differences with $P<0.05$ as statistically significant.

\section{Results}

\section{Fibroblast resistance to irradiation compared with HNSCC cells}

Radiotherapy utilizes the difference in radiosensitivity between target cancer cells and healthy cells; during radiotherapy, cancer cells are killed by irradiation, whereas other cells in the surrounding microenvironment survive. In particular, the major cells in the microenvironment, fibroblasts, survive and are involved in tissue remodeling after radiotherapy [12]. In the present study, we first assessed whether there was a difference in radiosensitivity between HNSCC cells and fibroblasts. When two HNSCC cell lines, SAS and FaDu, as well as a healthy fibroblast cell line were irradiated with 2, 5, and 10 Gy the survival rate of HNSCC cells decreased with irradiation in a dose-dependent manner relative to the survival of cells under nonirradiated conditions; it contrast, the fibroblasts did not show a significant decrease in survival rate (Fig. 1). This result suggests that fibroblasts are more resistant to radiation than HNSCC cells and may survive even after radiotherapy.

\section{Irradiation enhances the ability of fibroblasts to promote HNSCC cell migration}

Fibroblasts are known to contribute to cancer progression and to be important factors in cancer control in various solid cancers [13]. In addition, fibroblasts that survive after radiotherapy serve as a scaffold and contribute to cancer recurrence and metastasis $[5,6,14]$. In other words, irradiation enhances the ability of fibroblasts to promote the activity of cancer cells. In the present study, we investigated how irradiation affects the HNSCC-activating effect of fibroblasts. In a migration assay, HNSCC cells were seeded into semipermeable inserts and cocultured with or without irradiated or nonirradiated fibroblasts, which were seeded into the lower chamber. Our aim was to determine whether fibroblasts influence the migration of HNSCC cells and, if so, how irradiation might affect this ability. As shown in Fig. 2, the migration of HNSCC cells was enhanced even when they were cultured with nonirradiated fibroblasts, but this migration ability was further enhanced when cocultured with irradiated fibroblasts. Thus, irradiation seems to extend the ability of fibroblasts to promote HNSCC cell migration. Furthermore, the result 
suggests that the factor promoting the migration of HNSCC cells might be a liquid component secreted from fibroblasts.

\section{Irradiation increases the expression of IL-6 from fibroblasts}

The results shown in Fig. 2 suggest that fibroblasts use some humoral factor to induce migration of HNSCC cells and that the expression of this factor is increased by irradiation. Although various humoral factors are known to be involved in cancer progression, IL- 6 has recently been associated with various carcinomas including HNSCC. Because IL-6 elicits several tumor growth-promoting effects including promotion of cancer cell migration, it is also a promising therapeutic target $[15,16]$ (Johnson DE 2018; Choy 2020). Therefore, we investigated IL-6 as the potential factor secreted from fibroblasts; specifically, we assessed whether IL- 6 secretion was increased by irradiation. According to an ELISA, expression of IL6 in the culture media of irradiated (10 Gy) fibroblasts increased compared with its expression in media from nonirradiated fibroblasts (Fig. 3). This result indicates that irradiation induces IL-6 secretion from fibroblasts; it also suggests the possibility that IL- 6 is the factor that caused migration of HNSCC cells (see Fig. 2).

\section{IL-6 induces HNSCC cell migration}

To determine whether IL- 6 induces migration of HNSCC cells, we first confirmed whether HNSCC cells can be activated by IL- 6 . For IL- 6 to demonstrate its biological activities, the target cell must express IL- 6 receptor (IL-6R) and gp130 [17]. We confirmed that both SAS and FaDu HNSCC cell lines express IL-6R and gp130 via western blotting (Fig. 4A). This result suggests that HNSCC cells can be activated by an intracellular signal transduction pathway via exogenous IL-6. In addition, we conducted a migration assay using HNSCC cells with or without IL- 6 and found that HNSCC cell migration was increased in the presence of IL-6 (Fig. 4B).

\section{IL-6 plays a crucial role in the ability of fibroblasts to promote HNSCC migration}

We confirmed whether fibroblasts promote HNSCC cell migration using IL-6 secretion. For this purpose, we performed a HNSCC cell migration assay including coculturing with fibroblasts and with or without an IL-6 neutralizing antibody. Results showed that HNSCC cell migration was induced by coculturing with fibroblasts; however, this induction of HNSCC migration was significantly suppressed by the IL- 6 neutralizing antibody (Fig. 5). Thus, IL-6 secreted from fibroblasts is apparently involved in the enhancement of HNSCC cell migration.

\section{Discussion}

Invasion and distant metastasis are major characteristics of cancer that are associated with the progression of the disease and poor prognosis [18]. This is true for HNSCC, especially of distant metastases that are difficult to treat despite the development of therapeutic agents [19-21]. Thus, metastasis is clinically important in cancer treatment. It has been reported that the migration of cancer 
cells is essential in the process of metastasis. When cancer cells invade surrounding tissues, the cells migrate between tissues while destroying the surrounding tissues with enzymes. The migrating cells then infiltrate the vessels and become tumor cells that circulate throughout the body, creating metastatic lesions at distant locations. Thus, cell migration is an important first step in distant metastasis [22].

Previous studies have shown that irradiation contributes to increased distant metastases in many cancers, including head and neck cancers [23-25]. Therefore, the enhancement of cell migration by irradiation has attracted the attention of researchers. Various studies have shown hypoxia, epithelial-tomesenchymal transition, and specific cytokines as causes of irradiation-induced migration enhancement $[3,4]$. In particular, cytokines are secreted not only from cancer cells but also from the cells in the environment surrounding the cancer, and they are important for various tumor progression phenomena such as cancer cell invasion and the increase in circulating tumor cells $[8,10]$. Fibroblasts in the cancer microenvironment are known as cancer-associated fibroblasts; these are strongly associated with cancer cells and contribute to cancer progression including the progression of head and neck cancers [26, 27]. Under irradiation, fibroblasts are also important for cancer progression [28]. For example, in pancreatic and liver cancers under irradiation, fibroblasts increase the secretion of various humoral factors including cytokines such as bFGF, TNF-a, VEGF, IL-6, EGF, MMP-2, and MMP-9. These humoral factors contribute to cancer progression, including the migration and invasion of cancer cells after irradiation $[7,9]$. Thus, cytokines induced from fibroblasts by irradiation have important effects on the enhanced migration ability of cancer cells after irradiation.

In the present study, we showed that IL-6 secreted from fibroblasts is an important factor in the induction of HNSCC cell migration. IL- 6 has been primarily reported as a factor in inflammation, but it is also known to be involved in tumor progression in many cancers $[29,30]$. Therefore, its potential as a therapeutic target for cancer has been indicated; furthermore, the antitumor effect of inhibiting the JAK/STAT3 pathway, which is the main signaling pathway of IL-6, has been reported [30, 31]. In addition, IL6/JAK/STAT3 pathway inhibition has a synergistic antitumor effect with conventional cancer treatments, e.g., its effect is enhanced in combination with chemotherapy and radiotherapy [30, 32]. In particular, recent studies have reported that IL- 6 is associated with the effects of immune checkpoint inhibitor in the treatment of head and neck cancer. Consequently, IL-6 is a factor expected to become increasingly important in the treatment of HNSCC in the future.

\section{Conclusion}

The results of this study showed that fibroblasts survive irradiation and that irradiation induces expression of IL- 6 from fibroblasts. It was also found that irradiation enhances the ability of fibroblasts to promote the migration of HNSCC cells.

Moreover, it was confirmed that fibroblasts induce HNSCC cell migration through IL- 6 expression. Taken together, these results suggest the possibility that fibroblasts survive irradiation and enhance the promotion of HNSCC cell migration through IL- 6 secretion. To the best of our knowledge, we are the first 
to speculate on the mechanism by which irradiation promotes HNSCC cell migration. Thus, our results could contribute to the future development of therapeutics in HNSCC including the treatment of recurrence and metastasis after radiotherapy.

\section{Abbreviations}

DMEM Dulbecco's modified Eagle's medium

ELISA Enzyme-linked immunosorbent assay

FBS Fetal bovine serum

HNSCC Head and neck squamous cell carcinoma

SD Standard deviation

SDS Sodium dodecyl sulfate

\section{Declarations}

\section{Ethics approval and consent to participate}

Not applicable.

\section{Consent for publication}

Not applicable.

\section{Availability of data and material}

The datasets used and analyzed during the current study are available from the corresponding author on reasonable request.

\section{Competing interest}

The authors declare that the research was conducted in the absence of any commercial or financial relationships that could be construed as a potential conflict of interest.

\section{Funding}

The present study was supported by a Grant-in-Aid for Scientific Research (C) (grant no. 18K09337) from the Ministry of Education, Culture, Sports, Science and Technology, Japan.

\section{Author Contributions}


SS: conceptualization, design, and writing-original draft preparation. ST, YA, TE, and TK: data acquisition and interpretation. TY: supervision. All authors have read and approved the final manuscript.

\section{Acknowledgements}

Not applicable.

\section{References}

1. Ho JC, Phan J. Reirradiation of head and neck cancer using modern highly conformal techniques. Head and Neck. 2018;40:2078-93.

2. Siddiqui F, Movsas B. Management of Radiation Toxicity in Head and Neck Cancers. Seminars in Radiation Oncology. 2017;27:340-9. doi:10.1016/j.semradonc.2017.04.008.

3. Moncharmont C, Levy A, Guy JB, Falk AT, Guilbert M, Trone JC, et al. Radiation-enhanced cell migration/invasion process: A review. Critical Reviews in Oncology/Hematology. 2014;92:133-42. doi:10.1016/j.critrevonc.2014.05.006.

4. Vilalta M, Rafat M, Graves EE. Effects of radiation on metastasis and tumor cell migration. Cellular and Molecular Life Sciences. 2016;73:2999-3007. doi:10.1007/s00018-016-2210-5.

5. Straub JM, New J, Hamilton CD, Lominska C, Shnayder Y, Thomas SM. Radiation-induced fibrosis: mechanisms and implications for therapy. Journal of Cancer Research and Clinical Oncology. 2015;141:1985-94. doi:10.1007/s00432-015-1974-6.

6. Barker HE, Paget JTE, Khan AA, Harrington KJ. The tumour microenvironment after radiotherapy: Mechanisms of resistance and recurrence. Nature Reviews Cancer. 2015;15:409-25. doi:10.1038/nrc3958.

7. Ohuchida K, Mizumoto K, Murakami M, Qian LW, Sato N, Nagai E, et al. Radiation to Stromal Fibroblasts Increases Invasiveness of Pancreatic Cancer Cells through Tumor-Stromal Interactions. Cancer Research. 2004;64:3215-22. doi:10.1158/0008-5472.CAN-03-2464.

8. Kamochi N, Nakashima M, Aoki S, Uchihashi K, Sugihara H, Toda S, et al. Irradiated fibroblastinduced bystander effects on invasive growth of squamous cell carcinoma under cancer-stromal cell interaction. Cancer Science. 2008;99:2417-27. doi:10.1111/j.1349-7006.2008.00978.x.

9. Zhou LY, Wang ZM, Gao YB, Wang LY, Zeng ZC. Stimulation of hepatoma cell invasiveness and metastatic potential by proteins secreted from irradiated nonparenchymal cells. International Journal of Radiation Oncology Biology Physics. 2012;84:822-8. doi:10.1016/j.jijrobp.2012.01.011.

10. Bouchard G, Bouvette G, Therriault H, Bujold R, Saucier C, Paquette B. Pre-irradiation of mouse mammary gland stimulates cancer cell migration and development of lung metastases. British Journal of Cancer. 2013;109:1829-38. doi:10.1038/bjc.2013.502.

11. Ebbing EA, van der Zalm AP, Steins A, Creemers A, Hermsen S, Rentenaar R, et al. Stromal-derived interleukin 6 drives epithelial-to-mesenchymal transition and therapy resistance in esophageal 
adenocarcinoma. Proceedings of the National Academy of Sciences of the United States of America. 2019;116:2237-42. doi:10.1073/pnas.1820459116.

12. Baskar R, Lee KA, Yeo R, Yeoh KW. Cancer and radiation therapy: Current advances and future directions. International Journal of Medical Sciences. 2012;9:193-9. doi:10.7150/ijms.3635.

13. Liao Z, Tan ZW, Zhu P, Tan NS. Cancer-associated fibroblasts in tumor microenvironment Accomplices in tumor malignancy. Cellular Immunology. 2019;343. doi:10.1016/j.cellimm.2017.12.003.

14. Baskar R, Dai J, Wenlong N, Yeo R, Yeoh KW. Biological response of cancer cells to radiation treatment. Frontiers in Molecular Biosciences. 2014;1 NOV. doi:10.3389/fmolb.2014.00024.

15. Johnson DE, O'Keefe RA, Grandis JR. Targeting the IL-6/JAK/STAT3 signalling axis in cancer. Nature Reviews Clinical Oncology. 2018;15:234-48. doi:10.1038/nrclinonc.2018.8.

16. Choy EH, de Benedetti F, Takeuchi T, Hashizume M, John MR, Kishimoto T. Translating IL-6 biology into effective treatments. Nature Reviews Rheumatology. 2020;16:335-45. doi:10.1038/s41584-0200419-z.

17. Mihara M, Hashizume M, Yoshida H, Suzuki M, Shiina M. IL-6/IL-6 receptor system and its role in physiological and pathological conditions. Clinical Science. 2012;122:143-59. doi:10.1042/CS20110340.

18. Jiang WG, Sanders AJ, Katoh M, Ungefroren $H$, Gieseler F, Prince $M$, et al. Tissue invasion and metastasis: Molecular, biological and clinical perspectives. Seminars in Cancer Biology. 2015;35:S244-75. doi:10.1016/j.semcancer.2015.03.008.

19. Ferris RL, Blumenschein G, Fayette J, Guigay J, Colevas AD, Licitra L, et al. Nivolumab for Recurrent Squamous-Cell Carcinoma of the Head and Neck. New England Journal of Medicine. 2016;375:1856-67. doi:10.1056/NEJMoa1602252.

20. Burtness B, Harrington KJ, Greil R, Soulières D, Tahara M, de Castro G, et al. Pembrolizumab alone or with chemotherapy versus cetuximab with chemotherapy for recurrent or metastatic squamous cell carcinoma of the head and neck (KEYNOTE-048): a randomised, open-label, phase 3 study. The Lancet. 2019;394:1915-28. doi:10.1016/S0140-6736(19)32591-7.

21. Johnson DE, Burtness B, Leemans CR, Lui VWY, Bauman JE, Grandis JR. Head and neck squamous cell carcinoma. Nature Reviews Disease Primers. 2020;6. doi:10.1038/s41572-020-00224-3.

22. Entschladen F, Drell VI TL, Lang K, Joseph J, Zaenker KS. Tumour-cell migration, invasion, and metastasis: Navigation by neurotransmitters. Lancet Oncology. 2004;5:254-8. doi:10.1016/S14702045(04)01431-7.

23. Strong MS, Vaughan CW, Kayne HL, Aral IM, Ucmakli A, Feldman M, et al. A randomized trial of preoperative radiotherapy in cancer of the oropharynx and hypopharynx. The American Journal of Surgery. 1978;136:494-500.

24. Anderson P, Dische S. Local tumor control and the subsequent incidence of distant metastatic disease. International Journal of Radiation Oncology, Biology, Physics. 1981;7:1645-8. doi:10.1016/0360-3016(81)90186-3. 
25. Fagundes H, Perez CA, Grigsby PW, Lockett MA. Distant metastases after irradiation alone in carcinoma of the uterine cervix. International Journal of Radiation Oncology, Biology, Physics. 1992;24:197-204. doi:10.1016/0360-3016(92)90671-4.

26. Knops AM, South A, Rodeck U, Martinez-Outschoorn U, Harshyne LA, Johnson J, et al. CancerAssociated Fibroblast Density, Prognostic Characteristics, and Recurrence in Head and Neck Squamous Cell Carcinoma: A Meta-Analysis. Frontiers in Oncology. 2020;10. doi:10.3389/fonc.2020.565306.

27. Kalluri R. The biology and function of fibroblasts in cancer. Nature Reviews Cancer. 2016;16:582-98. doi:10.1038/nrc.2016.73.

28. Wang Z, Tang Y, Tan Y, Wei Q, Yu W. Cancer-associated fibroblasts in radiotherapy: challenges and new opportunities. doi:10.1186/s12964-019-0362-2.

29. Kumari N, Dwarakanath BS, Das A, Bhatt AN. Role of interleukin-6 in cancer progression and therapeutic resistance. Tumor Biology. 2016;37:11553-72. doi:10.1007/s13277-016-5098-7.

30. Jones SA, Jenkins BJ. Recent insights into targeting the IL- 6 cytokine family in inflammatory diseases and cancer. Nature Reviews Immunology. 2018;18:773-89. doi:10.1038/s41577-018-00667.

31. Rossi JF, Lu ZY, Jourdan M, Klein B. Interleukin- 6 as a therapeutic target. Clinical Cancer Research. 2015;21:1248-57. doi:10.1158/1078-0432.CCR-14-2291.

32. Long KB, Tooker G, Tooker E, Luque SL, Lee JW, Pan X, et al. IL6 receptor blockade enhances chemotherapy efficacy in pancreatic ductal adenocarcinoma. Molecular Cancer Therapeutics. 2017;16:1898-908. doi:10.1158/1535-7163.MCT-16-0899.

\section{Figures}


A

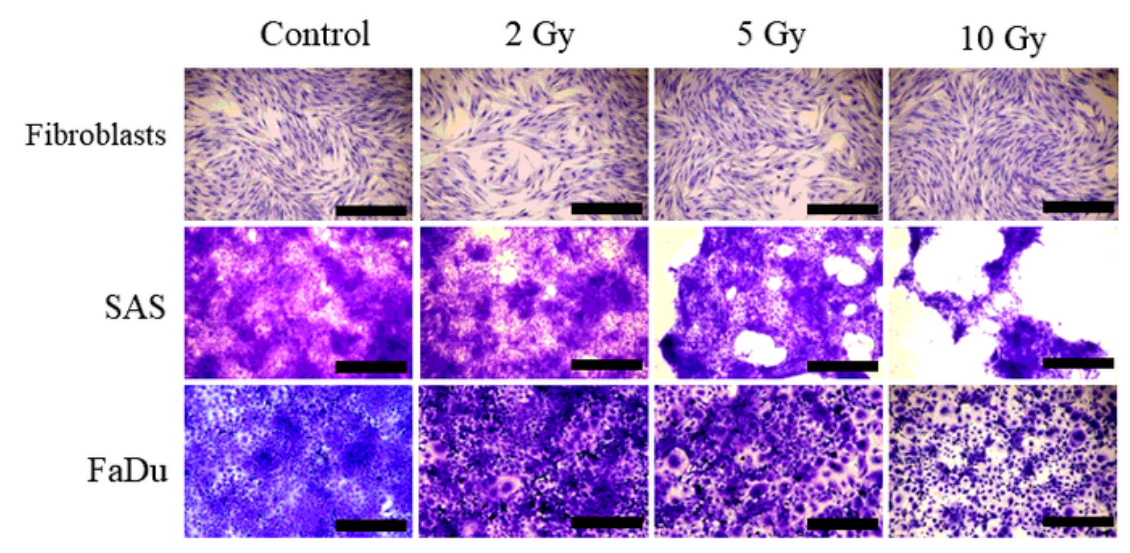

B

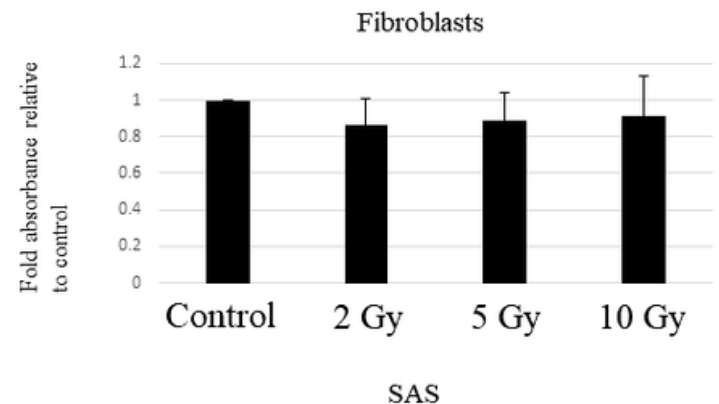

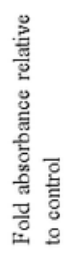

SAS
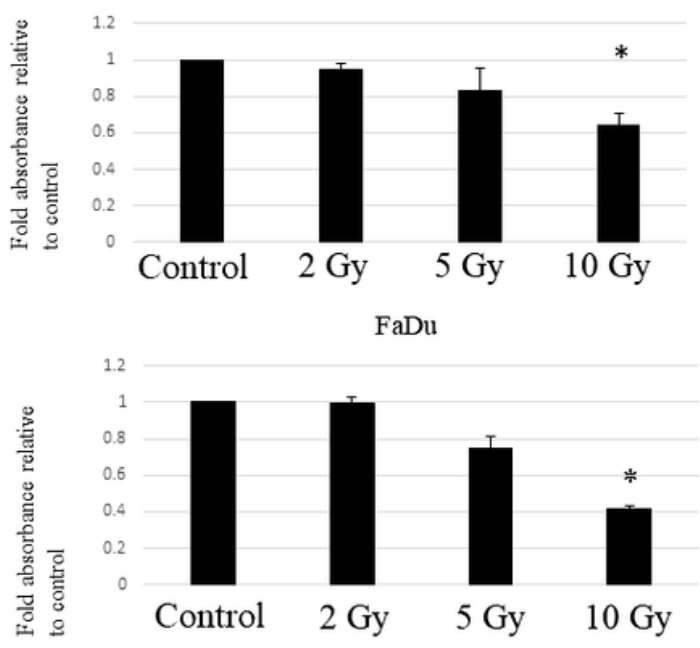

\section{Figure 1}

Effect of irradiation on the survival of HNSCC cells and fibroblasts. The viability of HNSCC cells and fibroblasts was evaluated by crystal violet staining. Each cell line was cultured to $80 \%$ confluence and irradiated at 2, 5, or $10 \mathrm{~Gy}$. At 6 days post-irradiation, the cells were fixed and stained by crystal violet. An unirradiated condition was used as a control in each cell line. Experiments were repeated three times. (A) Representative findings of cells observed with a light microscope. Scale bar: $1000 \mu \mathrm{m}$. (B) The cells were solubilized by $1 \%$ SDS and absorbance was measured at $550 \mathrm{~nm}$. Results are presented as fold-changes in absorbance relative to those under control conditions. Each experiment was repeated in triplicate and the data shown are means of three measurements with SD error bars. * $\mathrm{P}<0.05$. 
A

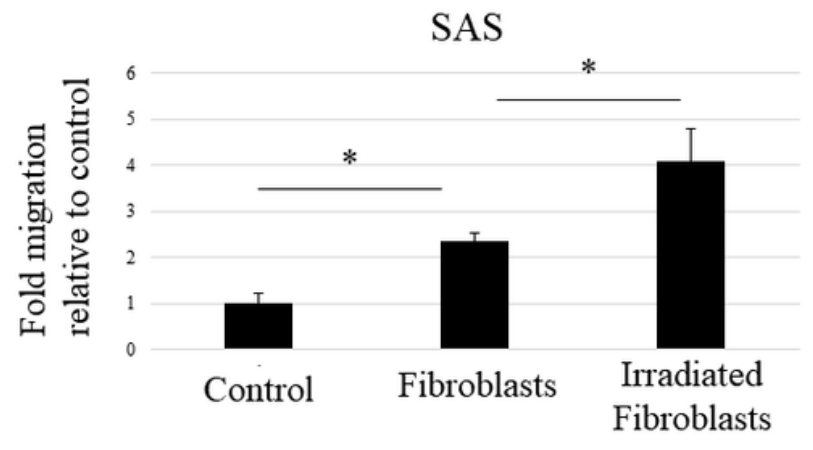

B

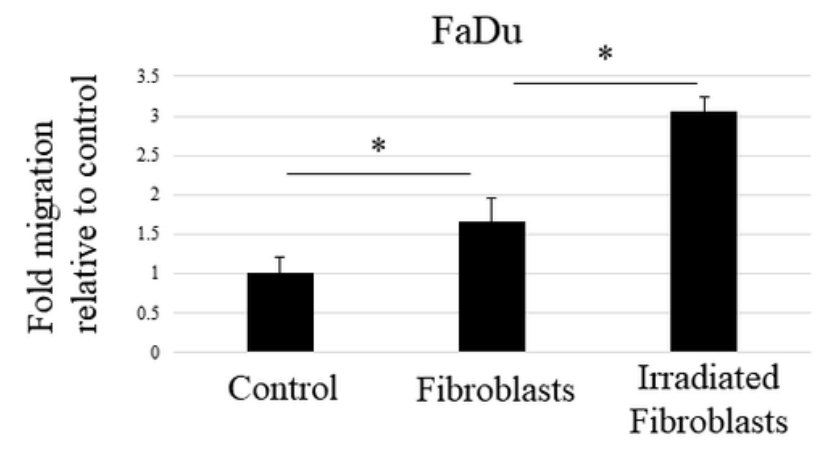

\section{Figure 2}

Irradiation enhances the ability of fibroblasts to promote HNSCC migration. A cell migration assay was performed with the HNSCC cell lines SAS (A) and FaDu (B). HNSCC cells were seeded into semipermeable inserts and cocultured with nonirradiated or irradiated fibroblasts seeded into the lower chamber. Results are expressed as fold-changes relative to HNSCC cells without coculture with fibroblasts (Control). HNSCC cell migration increased in the coculture with nonirradiated fibroblasts and was further increased by coculture with irradiated fibroblasts. The experiment was repeated three times and the data represent the means of three measurements with SD error bars. * $P<0.05$. 


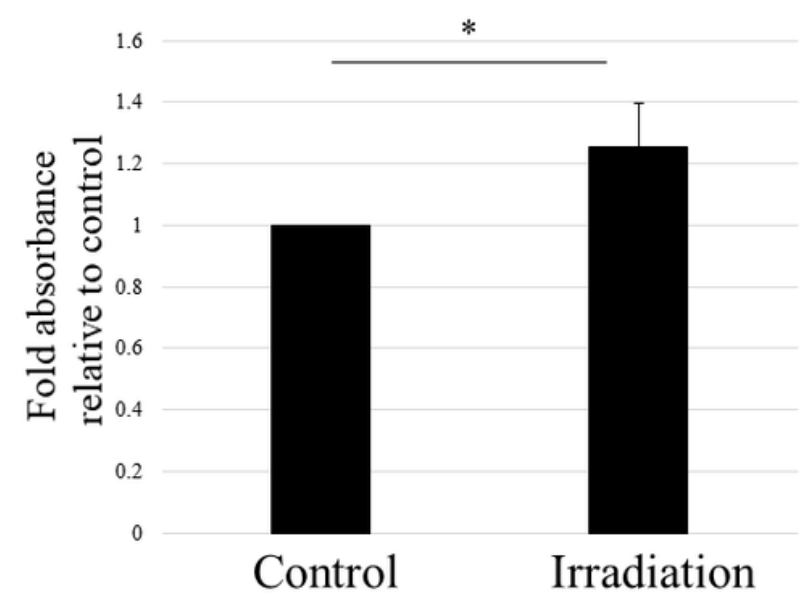

\section{Figure 3}

Irradiation increases expression of IL- 6 from fibroblasts. Expression of IL- 6 in culture media of fibroblasts with or without $10 \mathrm{~Gy}$ of irradiation was evaluated by ELISA. Results are expressed as fold-changes relative to fibroblasts without irradiation. Expression of IL-6 from fibroblasts was significantly increased with irradiation. The experiment was repeated in triplicate and the data represent the means of three measurements with SD error bars. * $P<0.05$. 
A

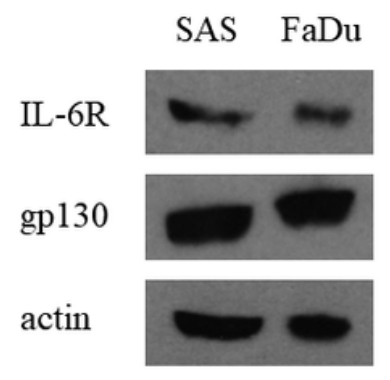

B

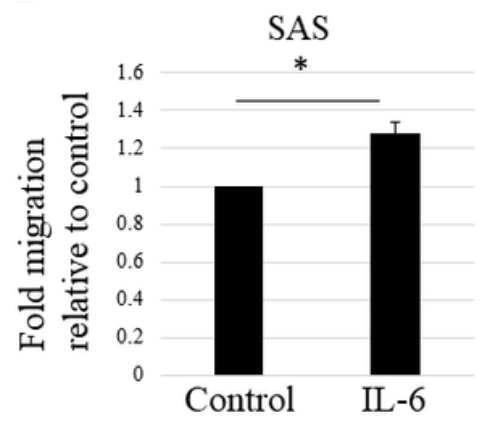

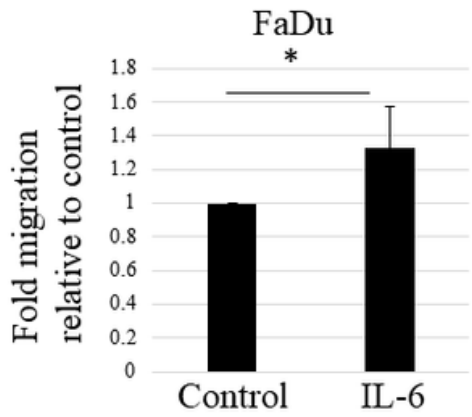

\section{Figure 4}

IL-6 induces HNSCC cell migration. (A) HNSCC cells express IL-6R and gp130. Expression of IL-6R and gp130 determined by western blot analysis in the HNSCC cell lines SAS and FaDu. The experiment was repeated three times with similar results. (B) IL-6 increases HNSCC cell migration. HNSCC cell lines, SAS, and FaDu, were subjected to a cell migration assay with or without $10 \mathrm{ng} / \mathrm{ml}$ of IL- 6 . HNSCC cell migration increased significantly with the addition of IL-6. The results are expressed as fold-changes relative to HNSCC cells without IL-6. Each experiment was repeated three times and the data shown are means of three measurements with SD error bars. * $\mathrm{P}<0.05$. 
A

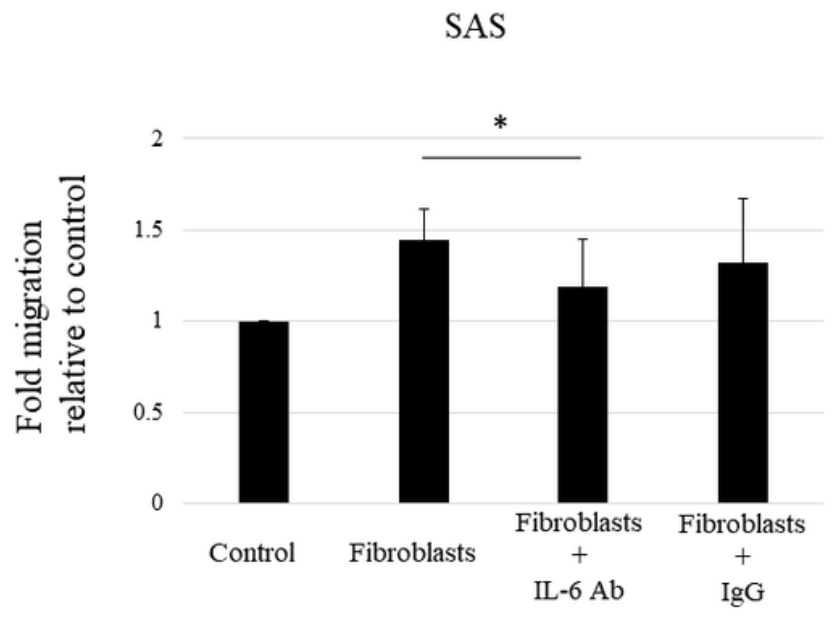

$\mathrm{B}$

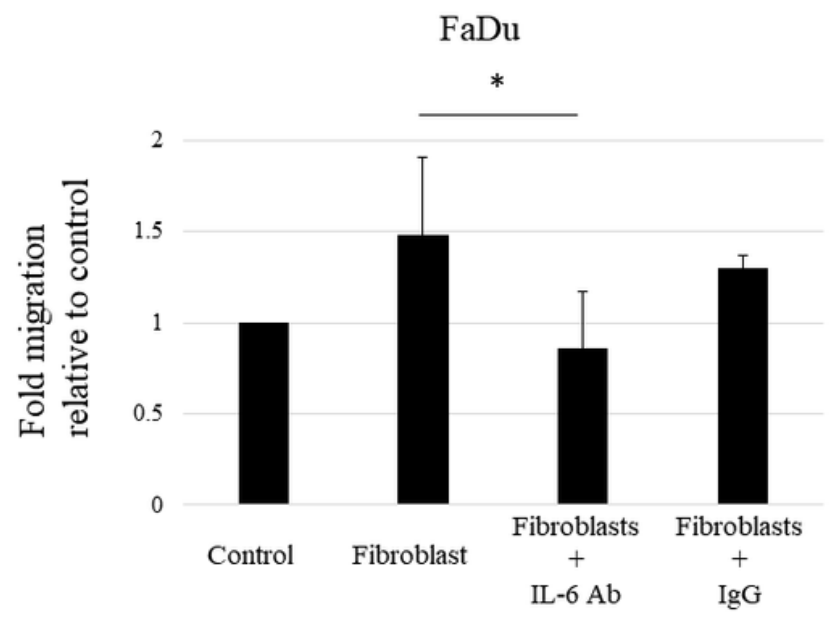

\section{Figure 5}

IL-6 plays a crucial role in the ability of fibroblasts to promote HNSCC migration. Neutralization of IL-6 abolishes the ability of fibroblasts to promote HNSCC migration. A cell migration assay of HNSCC cells, i.e., SAS (A) and FaDu (B), was performed with or without coculture with fibroblasts. In addition, cells were treated with $20 \mathrm{ng} / \mathrm{ml}$ of control monoclonal $\operatorname{lgG}(\mathrm{IgG})$ or anti-IL-6 mAb (IL-6 Ab) under coculture conditions. The results are expressed as fold-changes relative to HNSCC cells without coculture with fibroblasts. HNSCC cell migration increases by coculture with fibroblasts; however, this increase was prevented by treatment with anti-IL- $6 \mathrm{mAb}$. Each experiment was repeated in triplicate and the data represent the means of three measurements with SD error bars. ${ }^{*} P<0.05$. 